\title{
Establishment and characterization of a cell line from human adenoid cystic carcinoma of the lacrimal glands and a nude mouse transplantable model
}

\author{
TINGTING LIN*, LIMIN ZHU*, BEIQING ZHOU, LIANFENG XIE，JIANMEI LV, LIJIE DONG and YANJIN HE \\ Department of Orbital Disease and Oculoplastic Surgery, Tianjin Medical University Eye Hospital, \\ School of Optometry and Ophthalmology, TMU, Tianjin Medical University Eye Institute, Tianjin 300384, P.R. China
}

Received November 15, 2014; Accepted February 20, 2015

DOI: $10.3892 /$ or.2015.3925

\begin{abstract}
Using tissue block culture techniques, we established a new human tumor cell line derived from adenoid cystic carcinoma of the lacrimal glands (LACC-1). The LACC-1 cell line was successfully subcultured for more than 100 passages during the last two years. The outgrowth of cells was observed by day 5 after seeding, and then the cells were generated slowly. The first passage proceeded by day 32 , and the classical epithelioid cell colonies formed by day 69 after inoculation. After eight passages, homogeneous epithelioid tumor cells appeared when we combined continuous passage, mechanical scraping, repeated adherence, and dissociation methods to remove the fibroblast cells. LACC-1 cells appeared as a histologically solid pattern and continuous passage culture. The population doubling time was approximately $37.1 \mathrm{~h}$. LACC-1 cells appeared as an epithelioid monolayer culture on the cell culture flask and presented with a cobblestone-like appearance when they reached confluency. The nucleus was large and round with many abnormal mitoses. The nucleoplasm ratio was high. Multinucleated tumor giant cells appeared. LACC-1 cells showed a tendency to have overlapping growth without contact inhibition when the cell density continued to increase. Scanning electron microscopy (SEM) and transmission electron microscopy (TEM) showed that the LACC-1 cells were malignant tumor cells that were poorly differentiated. The surface of the LACC-1 cells exhibited affluent microvilli, protuberances and filopodia under SEM. The no. 84 genera-
\end{abstract}

Correspondence to: Professor Yanjin He, Tianjin Medical University Eye Hospital, School of Optometry and Ophthalmology, TMU, Tianjin Medical University Eye Institute, 251 Fu Kang Road, Nankai, Tianjin 300384, P.R. China

E-mail: yanjinhe1955@gmail.com

"Contributed equally

Key words: lacrimal glands, adenoid cystic carcinoma, cell line, biological characteristics, xenograft tumor, tumorigenicity tion LACC-1 cell line was inoculated subcutaneously into the subaxillary of nude mice and the tumorigenic potential was evident. The formation rate of the transplanted tumors was $100 \%$ at day 7 after inoculation. This finding showed that the LACC-1 cell line was malignant with tumorigenic ability. The xenograft tumors retained the same histological characteristics of a solid pattern as the LACC-1 original tumor after inoculation for 49 days. Under TEM observation, the xenograft tumor cells had the same ultrastructure as the LACC-1 cells. Immunohistochemical examination revealed the similarity of both cytoskeletal proteins (e.g., cytokeratin, vimentin, desmin and $\alpha$-SMA) and S-100 expression in the original tumor, LACC-1 cells and xenograft tumors. Immunoreactivity of these proteins was gradually decreased in these three tissues. Reverse transcription-polymerase chain reaction demonstrated that the xenograft tumors originated from the human. Based on these results, the LACC-1 cell line provides a useful model for studying the biological characteristics of human ACC of the lacrimal glands.

\section{Introduction}

Adenoid cystic carcinoma (ACC) is the most common malignant tumor of the lacrimal glands and is characterized by slow-growing, marked diffuse invasion into adjacent tissues and late onset of distant metastases (i.e., lungs, bones and other organs). Its prevalence is estimated to be $1.6 \%$ of all orbital tumors, $4.8 \%$ of all primary orbital neoplasms, and $25-30 \%$ of all epithelial neoplasms of the lacrimal gland (1-3). Patients with ACC of the lacrimal glands are difficult to cure due to the high recurrence rate, intracranial invasion and poor clinical outcome after long-term observation (4). However, ACC cell lines from human lacrimal glands have rarely been established. As no suitable cell lines or animal models exist, the characteristics of this tumor are not fully understood. Therefore, establishing an ACC cell line from human lacrimal glands (LACC-1) and an animal model is essential to analyze its biological behavior. In the present study, we describe the methods of establishment and the characteristics of a new ACC cell line isolated from human lacrimal glands and a heterotransplantation model of human lacrimal gland ACC into nude mice. 

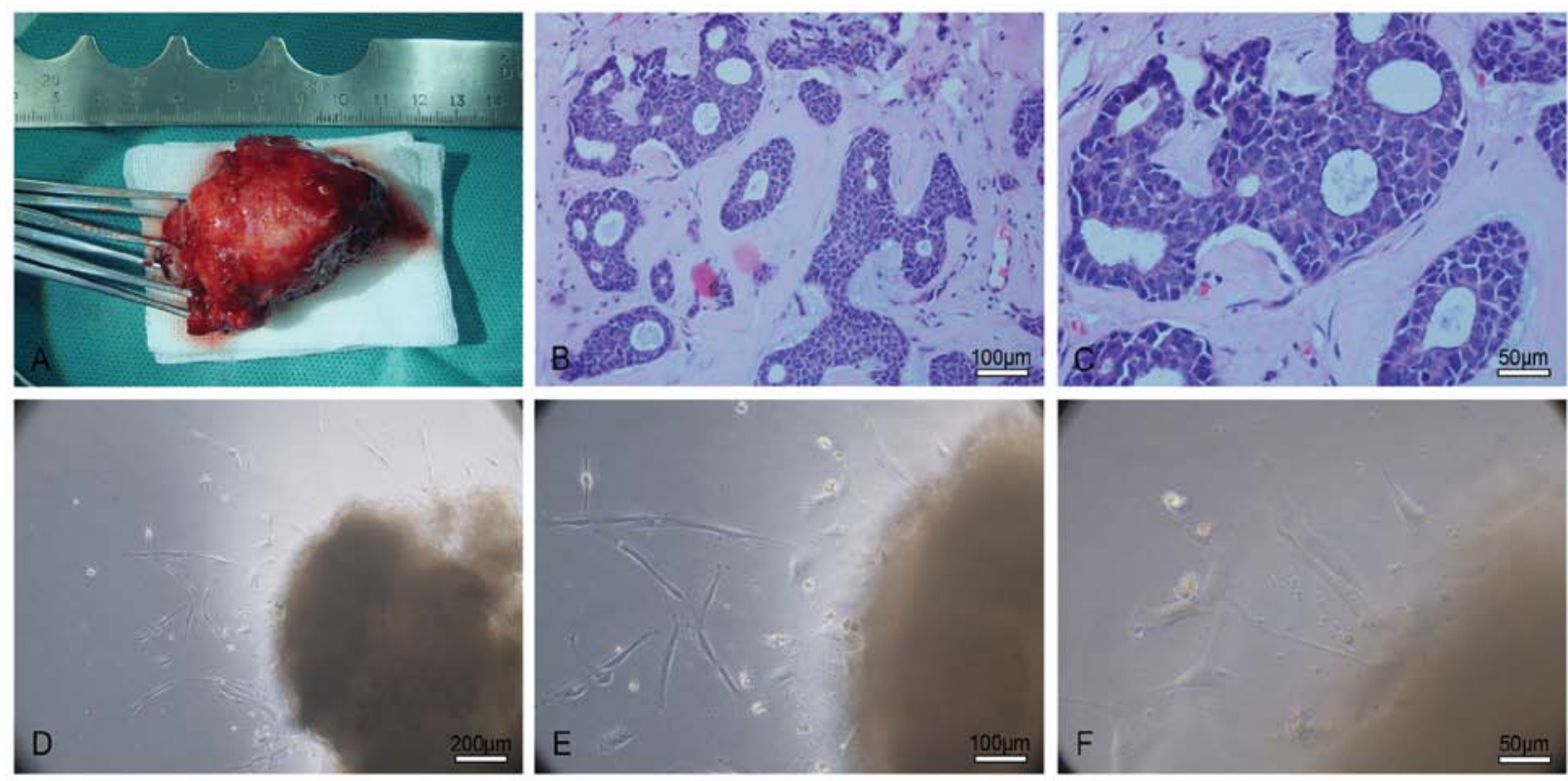

Figure 1. Establishment of the LACC-1 cell line. (A) The surgical specimen of a patient suffering from right lacrimal gland ACC. (B and C) H\&E staining. The tumor exhibits a solid pattern, and a cribriform structure is noted in the local area. Tumor cells are round or oval and have rich hyperchromatic nuclei and less cytoplasm. B: original magnification, x200; scale bar, $100 \mu \mathrm{m}$; C: Original magnification, x400; scale bar, $50 \mu \mathrm{m}$. (D-F) Approximately 5 days later, many cells grew from the edge of the explants. Most of the cells consist of fibroblasts and epithelioid tumor cells. D: Original magnification, $\mathrm{x} 100$; scale bar, $200 \mu$ m; E: original magnification, x200; scale bar, $100 \mu \mathrm{m}$; F: original magnification, $\mathrm{x} 400$; scale bar, $50 \mu \mathrm{m}$.

\section{Materials and methods}

Ethics statement. The use of tumor tissue for the present study was approved by the Human Research Ethics Committee of the Tianjin Medical University. The patient provided consent for her tumor samples to be used for scientific research purposes. Written informed consent was obtained from the patient. All of the animal experimental procedures were approved by the Institutional Animal Care and Use Committee (IACUC) of the Tianjin Medical University (permit no. SYXK 2009-0001), and were performed in compliance with the Association For Research In Vision And Ophthalmology Statement for the Use of Animals in Ophthalmic and Vision Research. Prior to sacrifice, general anesthesia was induced by intraperitoneal injection of chloral hydrate to alleviate pain. Mice were sacrificed using the cervical dislocation method.

Establishment of a new cell line. Tumor tissue was obtained from surgical material of a 35-year-old female patient with a solid pattern of ACC on the right lacrimal gland (Fig. 1A-C). The tumor tissue was placed into precooled RPMI-1640 medium (Hyclone Laboratories, Logan, UT, USA) immediately after being excised and then transported to the laboratory. The tumor was rinsed two to three times with RPMI-1640 medium containing $100 \mathrm{U} / \mathrm{ml}$ penicillin and $100 \mu \mathrm{g} / \mathrm{ml}$ streptomycin (Hyclone Laboratories) under sterile conditions. All necrotic tissue, fat, vessels and conjunctival tissue were removed. The tumor was cut into $0.5-1 \mathrm{~mm}^{3}$ pieces with scissors and seeded on the bottom of $25-\mathrm{cm}$ cell culture flasks, which had been pretreated with serum. The distance between each tumor piece was $\sim 5 \mathrm{~mm}$. Each flask contained 25-30 tumor pieces. The tumor pieces were cultured in RPMI-1640 medium supplemented with $20 \%$ fetal bovine serum (FBS; HyClone Laboratories), 2 mM L-glutamine (Gibco, Grand Island, NY,
USA), $100 \mathrm{U} / \mathrm{ml}$ penicillin and $100 \mu \mathrm{g} / \mathrm{ml}$ streptomycin as growth medium at $37^{\circ} \mathrm{C}$ in a $5 \% \mathrm{CO}_{2}$ incubator (Thermo Fisher Scientific, Waltham, MA, USA, model: 3111). Approximately 5 days later, many cells grew from the edge of the explants. Most of these cells contained fibroblasts and epithelioid tumor cells (Fig. 1D-F). Approximately 32 days later, the cells reached $80 \%$ confluency and were subcultured after dissociation with $0.25 \%$ trypsin and $0.53 \mathrm{mM}$ EDTA mixture (Beijing Solarbio Science \& Technology Co., Ltd., Beijing, China). In order to discard the fibroblasts and purified tumor cells, we combined continuous passage, mechanical scraping, repeated adherence and dissociation methods. ACC cell clones appeared 69 days later. The medium was replaced every two to three days. The ACC cells were subcultured when they reached $80 \%$ confluency.

Cell morphology. Tumor pieces were observed under a phase contrast microscope (Olympus IX70; Olympus Corp., Tokyo, Japan) every day.

Transmission electron microscopy (TEM) observation. When purified tumor cells were subcultured at the exponential stage, they were seeded in 75-cm cell culture flasks (Corning, Acton, MA, USA). The medium was replaced one day before the cells reached $100 \%$ confluency. The cells were collected by scraping and then the fixative was added. The cells were centrifuged at 1,000 rpm for $10 \mathrm{~min}$, and the supernatant was discarded carefully. The fixative was added again, and the cells were placed at $4^{\circ} \mathrm{C}$ for $2 \mathrm{~h}$. The fixative was replaced with fresh buffer every $10 \mathrm{~min}$ for three times. The cell pellets were preserved in fresh buffer at $4^{\circ} \mathrm{C}$ and sent to the Neurosurgery Institute in Beijing the next day. The institute was responsible for embedding, sectioning and conducting the TEM observation. 
Scanning electron microscopy (SEM) observation. Purified tumor cells were seeded on slides in 6-well plates (Corning) and incubated at $37^{\circ} \mathrm{C}$ in a $5 \% \mathrm{CO}_{2}$ incubator when they grew at the logarithmic phase. The size of the slide was $10 \mathrm{~mm}$ x $10 \mathrm{~mm}$. The medium was changed one day before the cells reached $100 \%$ confluency. The following day, the medium was extracted and gently rinsed with phosphate-buffered solution $(0.01 \mathrm{M}$ PBS; Gibco) for three times. The electron microscopy fixative was added and preserved at $4^{\circ} \mathrm{C}$ in the refrigerator. The next day, the samples were sent to the Neurosurgery Institute in Beijing. The institute was in charge of dehydration, coating the film and conducting the SEM observation.

Immunocytochemical (IHC) detection of protein expression. Purified tumor cells were plated on $25 \mathrm{~mm} \times 75 \mathrm{~mm}$ glass slides at a density of $5 \times 10^{4} \mathrm{cells} / \mathrm{ml}$ in a $10-\mathrm{cm}$ dish (Corning) and incubated at $37^{\circ} \mathrm{C}$ in a $5 \% \mathrm{CO}_{2}$ incubator. The medium was discarded when the tumor cells reached $70-80 \%$ confluency. The cells were rinsed with $0.01 \mathrm{M}$ PBS (pH 7.2 to 7.4) three times and fixed in 4\% paraformaldehyde (analytical grade; Yingdaxigui Chemical Reagent Factory, Tianjin, China) for $10 \mathrm{~min}$ at room temperature. The cells were separately incubated with primary antibodies against S-100 (rabbit polyclonal anti-S-100 protein; Invitrogen Carlsbad, CA, USA), cytokeratin (CK; rabbit anti-cytokeratin Pan; Invitrogen), vimentin (mouse anti-vimentin, clone V9; Invitrogen), $\alpha$-SMA (actin, smooth muscle, mouse monoclonal antibody, clone 1A4; Invitrogen) and desmin (mouse anti-desmin; Invitrogen) overnight at $4^{\circ} \mathrm{C}$ in a humidified box. Streptavidin-peroxidase immunohistochemistry detection kit (SP-9000 Histostain ${ }^{\mathrm{TM}}$-Plus kit; Zymed Laboratories, San Francisco, CA, USA) was used to detect protein expression. The brown color was developed using DAB kit (Beijing Zhongshan Golden Bridge Biotechnology Co., Ltd, Beijing, China) and observed by light microscopy (Olympus BX51; Olympus). The primary antibody of the negative controls was omitted and replaced wtih PBS.

Animals. Ten 4- to 5-week-old female nude mice (BALB/c $\mathrm{nu} / \mathrm{nu}$ ), which were purchased from the Beijing Academy of Military Medical Sciences Laboratory Animal Center, were used for the animal experiments. The mice weighed 18-20 g and were maintained under specific pathogen-free (SPF) conditions. The animals had free access to food and water and were maintained under a $12-\mathrm{h}: 12$-h light-dark cycle at $22-25^{\circ} \mathrm{C}$. The no. 84 generation LACC-1 cells growing at a logarithmic stage were trypsinized, washed twice and suspended in PBS. Then, cells $\left(1 \times 10^{7} / \mathrm{ml}\right)$ suspended in $0.1 \mathrm{ml}$ PBS were inoculated subcutaneously into the subaxillary of the nude mice. The longest length (L) and shortest width (W) of each xenograft tumor forming at the transplanted site were measured by an electronic caliper (Shanghai Shen Han Measuring Tools Co., Ltd., Shanghai, China; 0-150 mm) every day. Tumor volume (V) of the xenograft tumors was calculated using the formula: $\mathrm{V}=\left(\mathrm{LxW}^{2}\right) / 2$ and the tumor growth curve was constructed. At day 14 after the xenograft tumors were implanted, TEM observation was conducted. At 14, 28, 35, 42 and 49 days after implantation, two mice were sacrificed. Before sacrifice, general anesthesia was induced by intraperitoneal injection of chloral hydrate to alleviate pain. The lungs, livers and alar lymph nodes were excised for hematoxylin and eosin (H\&E) staining to observe tumor metastasis. The heterotransplanted tumors were removed for H\&E and immunohistochemistry (IHC) studies. Reverse transcription-polymerase chain reaction (RT-PCR) was performed on the tumors to detect human and mouse $\beta$-actin genes to confirm that they originated from human tissue and not from mouse tissue.

$H \& E$ staining and $I H C$. The heterotransplanted tumors and the lungs, livers and alar lymph nodes of the mice were fixed in 10\% formalin (analytical grade; Yingdaxigui Chemical Reagent Factory) for $24 \mathrm{~h}$ at room temperature, and gradient ethanol (analytical grade; Tianjin Fengchuan Chemical Reagent Science and Technology Co., Ltd., Tianjin, China) and dehydrated xylene (analytical grade; Tianjin Fengchuan Chemical Reagent Science and Technology), made transparent, embedded in paraffin (Shanghai Hualing Recovery Appliance Factory, Shanghai, China), and cut into $4-\mu \mathrm{m}$ sections. The sections were stained with $\mathrm{H} \& \mathrm{E}$ for histological examination. The tumor tissue sections of the LACC-1 cells grown in nude mice were incubated with primary antibodies against S-100, CK, vimentin, $\alpha$-SMA and desmin overnight at $4^{\circ} \mathrm{C}$ in a humidified box. Streptavidin-peroxidase IHC detection kit was used to detect protein expression. The brown color was developed using DAB kit and observed by light microscopy. The primary antibody of the negative controls was omitted and replaced by PBS.

RT-PCR analysis. Total-RNAs from the xenograft tumors, LACC-1 cells and nude mouse livers were extracted using TRIzol (Invitrogen). PCR of human $\beta$-actin cDNA was conducted using a sense primer, 5'-TGACGGGGTCACCCA CACTGTGCCCATCTA-3' and an antisense primer, 5'-CTA GAAGCATTTGCGGTGGACGATGGAGGG- 3 ', at $95^{\circ} \mathrm{C}$ for $4 \mathrm{~min}$ and for 35 cycles at $95^{\circ} \mathrm{C}$ for $30 \mathrm{sec}, 66^{\circ} \mathrm{C}$ for $30 \mathrm{sec}$, $72^{\circ} \mathrm{C}$ for $1 \mathrm{~min}$ and $72^{\circ} \mathrm{C}$ for $7 \mathrm{~min}$. The primers for mouse $\beta$-actin were sense, 5'-GATGACGATATCGCTGCGCTG-3', and antisense, 5'-GTACGACCAGAGGCATACAGG-3', and PCR was conducted at $95^{\circ} \mathrm{C}$ for $4 \mathrm{~min}$ and for 35 cycles at $95^{\circ} \mathrm{C}$ for $30 \mathrm{sec}, 58^{\circ} \mathrm{C}$ for $30 \mathrm{sec} 72^{\circ} \mathrm{C}$ for $1 \mathrm{~min}$ and $72^{\circ} \mathrm{C}$ for $7 \mathrm{~min}$. PCR products were electrophoresed on agarose gels (Biowest; Gene Tech Co., Ltd., Shanghai, China), stained with ethidium bromide and visualized under ultraviolet illumination (BioSpectrum AC Chemi HR 410; UVP LLC, Upland, CA, USA).

\section{Results}

Establishment of a new ACC cell line. This newly established cell line called LACC-1 was subcultured in $>100$ passages during the last two years. After eight passages, homogeneous epithelioid tumor cells appeared when we combined continuous passage, mechanical scraping, repeated adherence, and dissociation methods to remove the fibroblast cells. LACC-1 cells had long elliptical, triangular, spindle-like, cuboidal and polygonal morphologies when they were single or did not reach full confluency. They appeared as an epithelioid monolayer culture on a cell culture flask and presented with a cobblestone-like appearance when they reached confluence. The nuclei were large and round with many abnormal mitoses. The nucleoplasm ratio was high. Multinucleated giant cells 


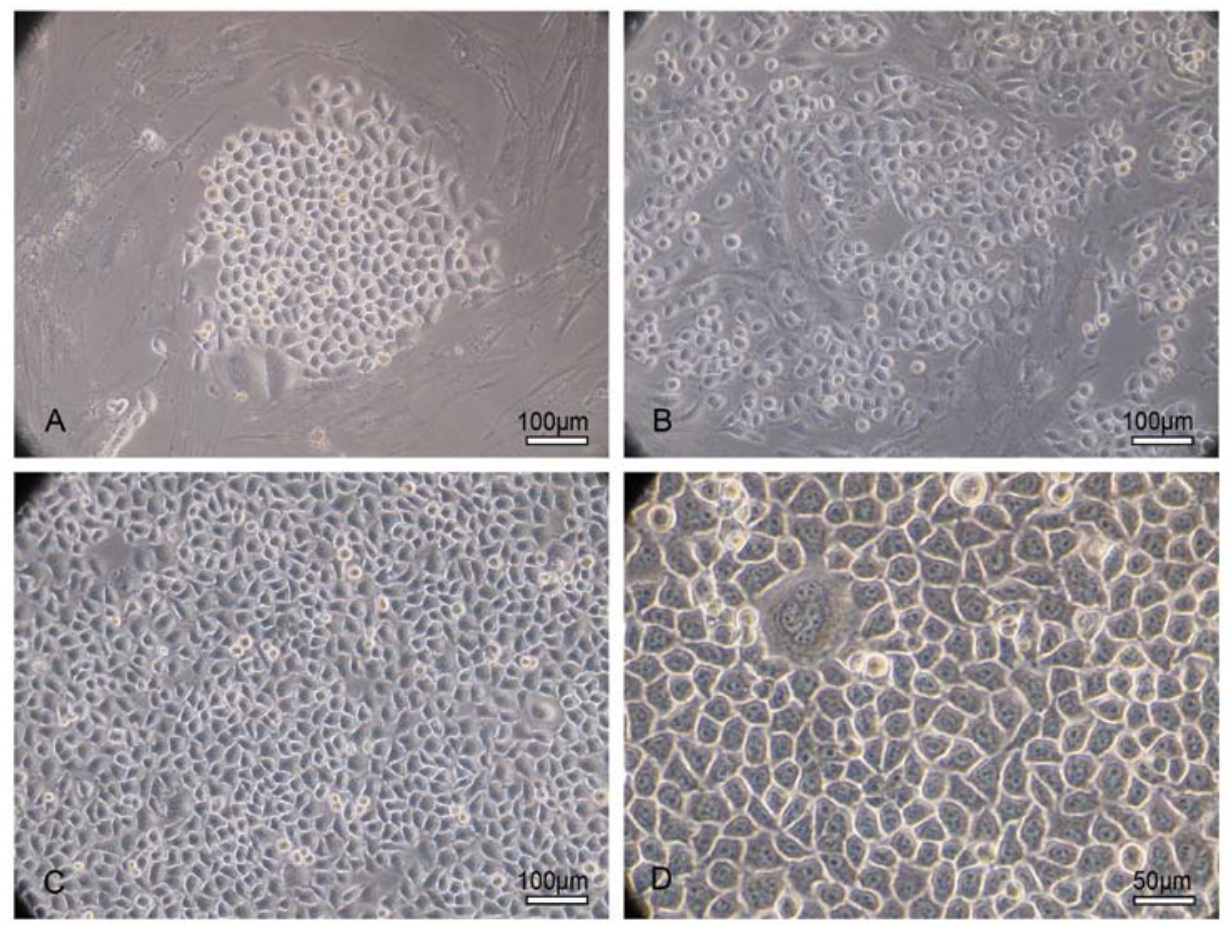

Figure 2. Morphology of the LACC-1 cells. (A) Phase contrast photomicrograph of epithelioid tumor cell clones in culture observed by day 72 after seeding. The cell clone presents with a cobblestone-like appearance and is surrounded by fibroblasts that grow in a whirl. Original magnification, $\mathrm{x} 200$; scale bar, $100 \mu \mathrm{m}$. (B) At 2-7 passages, the tumor cells become the dominant cells, and the fibroblasts are fewer. Original magnification, $\mathrm{x} 200$; scale bar, $100 \mu \mathrm{m}$. (C) After 8 passages, homogeneous epithelioid tumor cells appear. Fibroblast cells are absent. LACC cells exhibit triangular, cuboidal and polygonal morphologies as they reach full confluency. Original magnification, x200; scale bar, $100 \mu \mathrm{m}$. (D) After 8 passages, the cells grow as an epithelioid monolayer and present with a cobblestone-like appearance when they reached confluency. The nuclei are large and round with many abnormal mitoses. The nucleoplasm ratio is high. Multinuclear giant cells appear. LACC cells show a tendency to have overlapping growth without contact inhibition as the cell density increases. Original magnification, $\mathrm{x} 400$; scale bar, $50 \mu \mathrm{m}$.

appeared. LACC-1 cells showed a tendency to have overlapping growth without contact inhibition when the cell density continued to increase (Fig. 2).

As shown in the cell growth curve, the population doubling time was $\sim 37.1 \mathrm{~h}$ (Fig. 3).

TEM observation. Poorly differentiated LACC-1 cells were different in size and shape. Mitosis and multinucleated tumor giant cells were common. Cytoplasm was small, and cell nuclei were large and markedly atypical. The karyoplasmic ratio was reversed. One to several nucleoli and many chromatins in the nuclei were found. Euchromatin was abundant, and heterochromatin was highly marginated along the nuclear membrane. Irregular dents accompanied by occasional intranuclear pseudoinclusions were found on the surface of most nuclear membranes. In the cytoplasm, spherical secretory granules, which had uniform high-electron density, appeared. Many free ribosomes were found in the cytoplasm. Large ribosomes also appeared. The development of the rough endoplasmic reticulum was not balanced in different cells. Golgi complexes were occasionally visible. Other organelles were poorly developed. Lacunas were observed between cancer cells. The free surface of the cells was covered with many microvilli, which were uneven in length. Cancer cells were connected by a tight junction instead of a typical desmosome junction (Fig. 4).

SEM observation. The LACC-1 cells were mostly polygonal but appeared in other sizes and shapes. Abundant microvilli

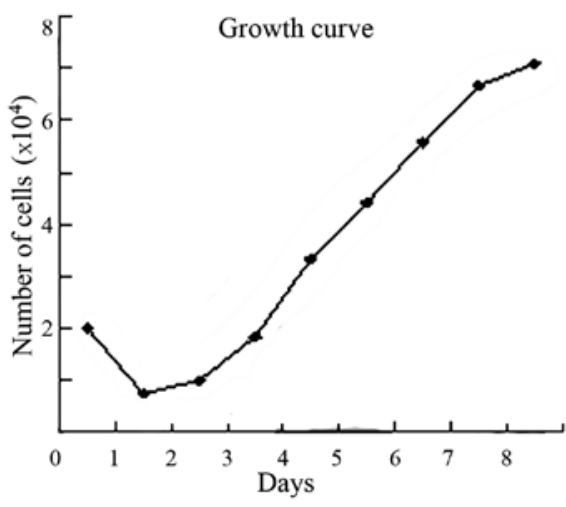

Figure 3. Cell growth curve of the LACC-1 cells.

were apparent on the surface of the cells. Cellular processes looked like lamellar or vesicular structures. Adjacent cell processes were interconnected. In the mitotic phase, the cells were apophysis. Numerous filopodia appeared on the surface of the cancer cells except dense microvilli. Filopodia appeared as a multistage branch that could support the apophysis of the cancer cells. Adjacent cells were connected by active filopodia (Fig. 5).

Animals. Two days after subcutaneous injection of $1 \times 10^{6}$ cells, some of the mice developed nodules under the subaxillary. All 10 mice developed solid tumors at 7 days after inoculation. The formation rate of the transplanted tumor was $100 \%$. The 

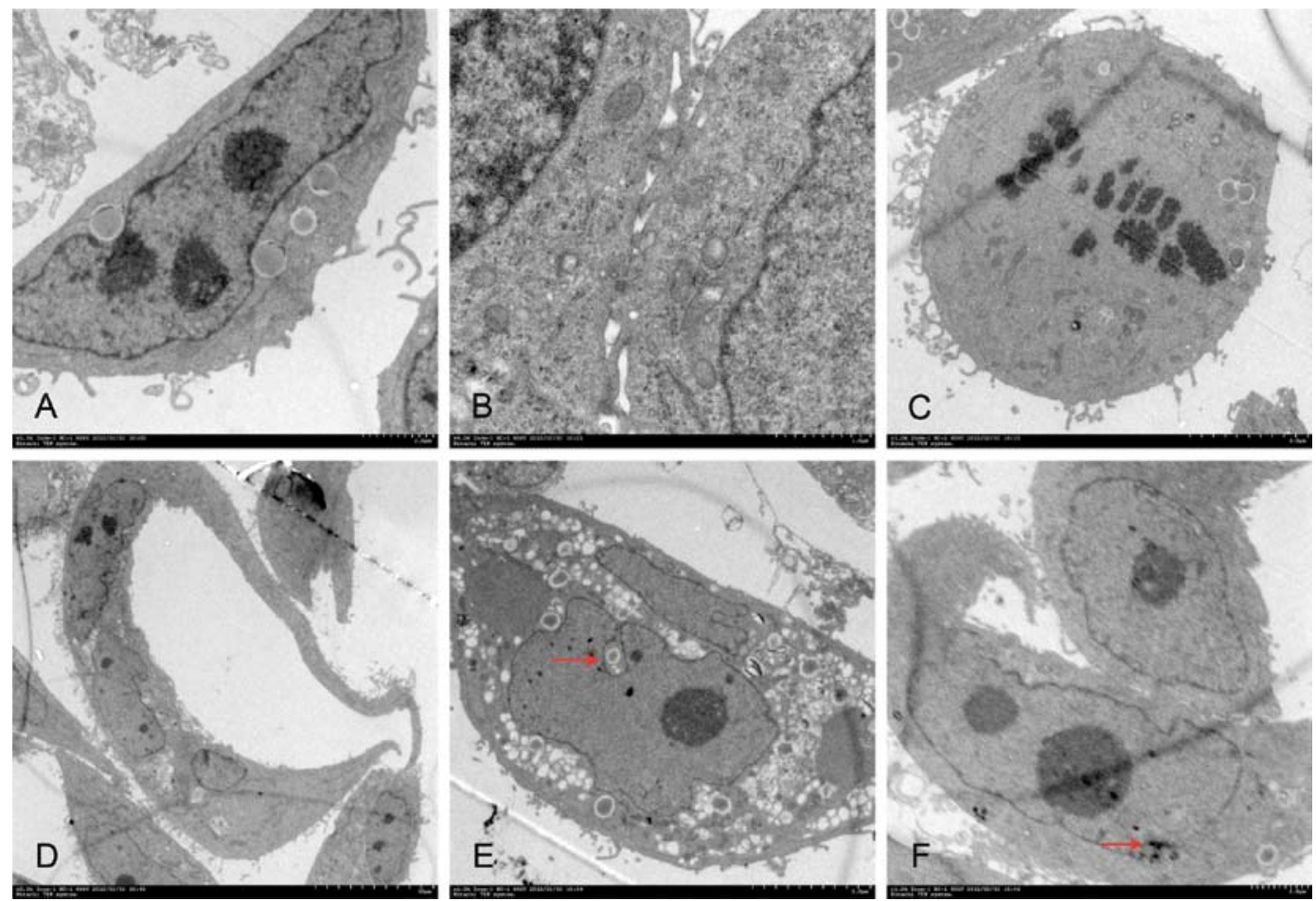

Figure 4. Transmission electron microscopic observation. (A) The karyoplasmic ratio is reversed. Three large nuclei appear. Original magnification, $\mathrm{x} 2.5 \mathrm{k}$; scale bar, $2.0 \mu \mathrm{m}$. (B) Cancer cells are connected by a tight junction. Original magnification, $\mathrm{x} 4 \mathrm{k}$; scale bar, $1.0 \mu \mathrm{m}$. (C) The cell undergoes division, and its free surface is covered with microvilli. Original magnification, $\mathrm{x} 1 \mathrm{k}$; scale bar, $5.0 \mu \mathrm{m}$. (D) Multinucleated tumor giant cells. Original magnification, $\mathrm{x} 0.5 \mathrm{k}$; scale bar, $10.0 \mu \mathrm{m}$. (E) Intranuclear pseudoinclusion (red arrow). Original magnification, x1k; scale bar, $5.0 \mu \mathrm{m}$. (F) Huge ribosomes appear (red arrow). Original magnification, $\mathrm{x} 1.2 \mathrm{k}$; scale bar, $2.0 \mu \mathrm{m}$.
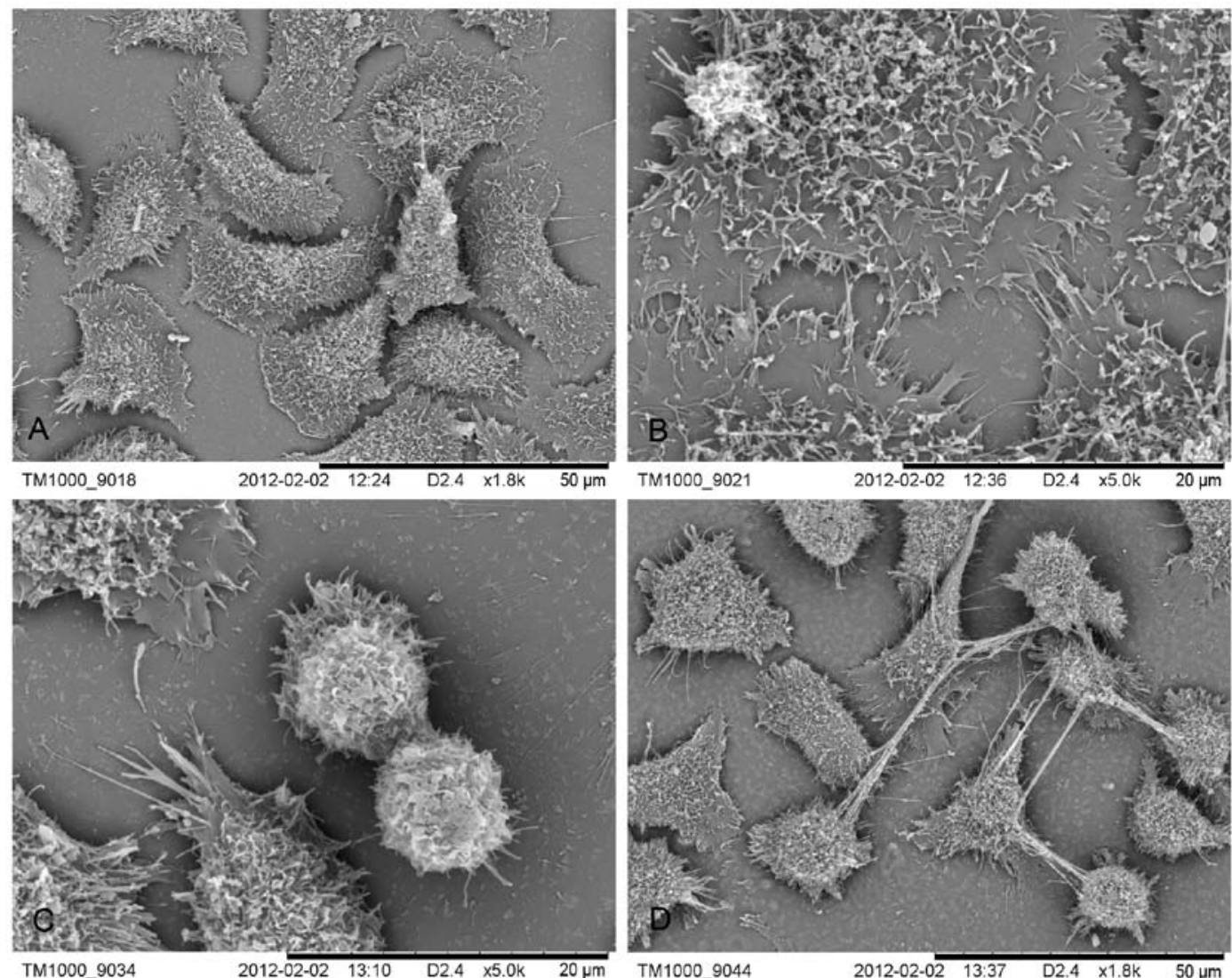

Figure 5. Scanning electron microscopic observation. (A) Many microvilli are noted on the surface of the cells. The filopodia appear as a multistage branch. Adjacent cell processes are interconnected. Original magnification, x1.8k; scale bar, $50 \mu \mathrm{m}$. (B) Adjacent cells are interconnected. Original magnification, x5.0k; scale bar, $20 \mu \mathrm{m}$. (C) Cell division phase. Original magnification, x5.0k; scale bar, $20 \mu \mathrm{m}$. (D) Cell interconnection exists not only between adjacent cancer cells but also among cells far from each other. Original magnification, x1.8k; scale bar, $50 \mu \mathrm{m}$. 


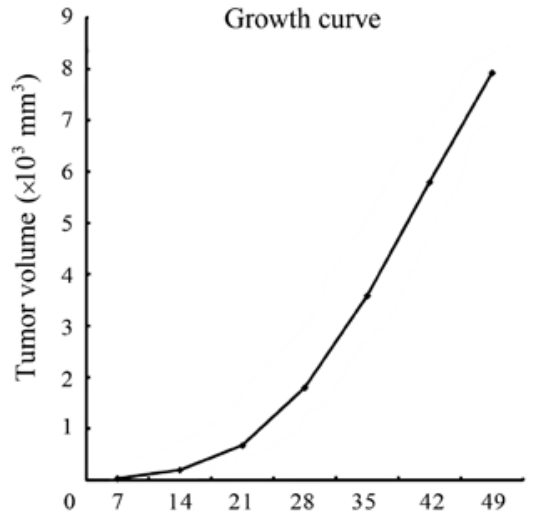

Figure 6. Growth curve of the xenograft tumor volume.

tumor volumes were observed at $7,14,21,28,35,42$ and 49 days after injection, and the growth curve was determined (Fig. 6). The transplanted tumors grew faster in the first three weeks after injection.

Fourteen days after injection, the xenograft tumors became round or oval and covered with a capsule. The graft tumors exhibited no adhesion to the adjacent tissue. The texture of the tumor was medium. The color of the section was pale. At 49 days after injection, the xenograft tumors were still covered with a capsule. They did not adhere to the viscera but intruded into the chest wall and thoracic cavity. No lung, liver and axillary lymph node metastases occurred (Fig. 7).

The xenograft tumors were excised on day 14, 28, 35, 42 and 49 after injection. The main structure of the tumors was the same but more necrotic tissue was present in the center of the tumor as time passed. As observed under a light microscope, the tumors were covered with a thin connective tissue. The tumors exhibited mostly a solid pattern and mucoid degeneration occurred in the local area. Tumor cells were round or oval. They had large hyperchromatic nuclei and less cytoplasm. Mitosis and multinucleated giant tumor cells were commonly noted (Fig. 8).

The xenograft tumor cells had the same ultrastructure as the LACC-1 cells under TEM observation. However, the tumor cells were mostly connected by a gap junction instead of a tight junction. Some dysplastic desmosomes appeared occasionally. A small amount of microvilli covered the free surface of the tumor cells. No form of mature glandular tubes was noted (Fig. 9).

Immunocytochemical detection of protein expression. The immunohistochemical examinations revealed the similarity of cytoskeletal protein (e.g., CK, vimentin, $\alpha$-SMA and desmin)
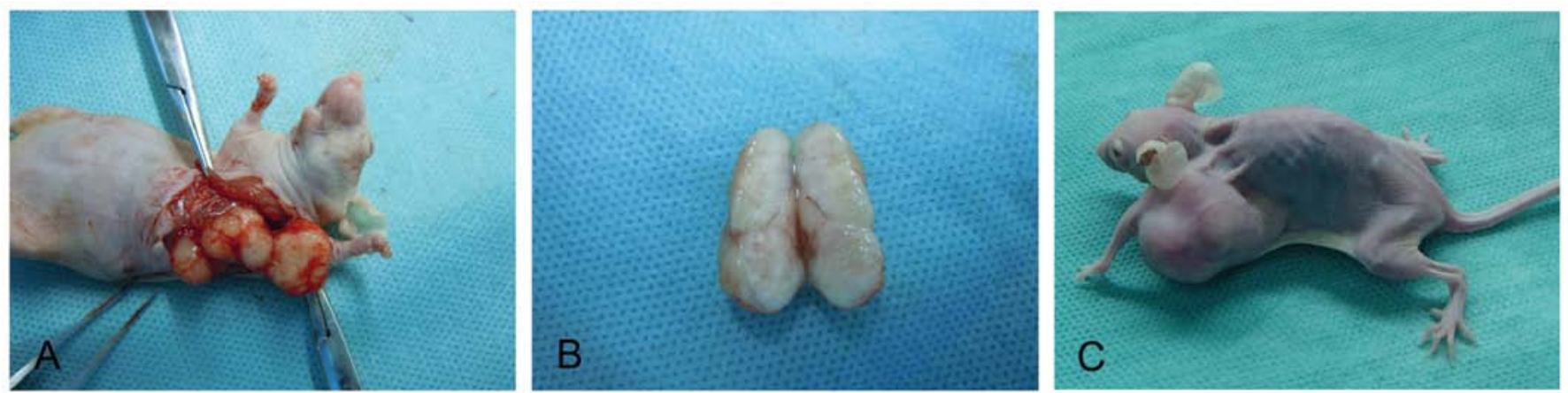

Figure 7. Morphology of the xenograft tumors. (A) Thirty-five days after injection. The xenograft tumors are round or oval and covered with thin capsules. (B) Thirty-five days after injection. The texture of the graft tumor is medium and the color of the section is pale. (C) Forty-nine days after injection.
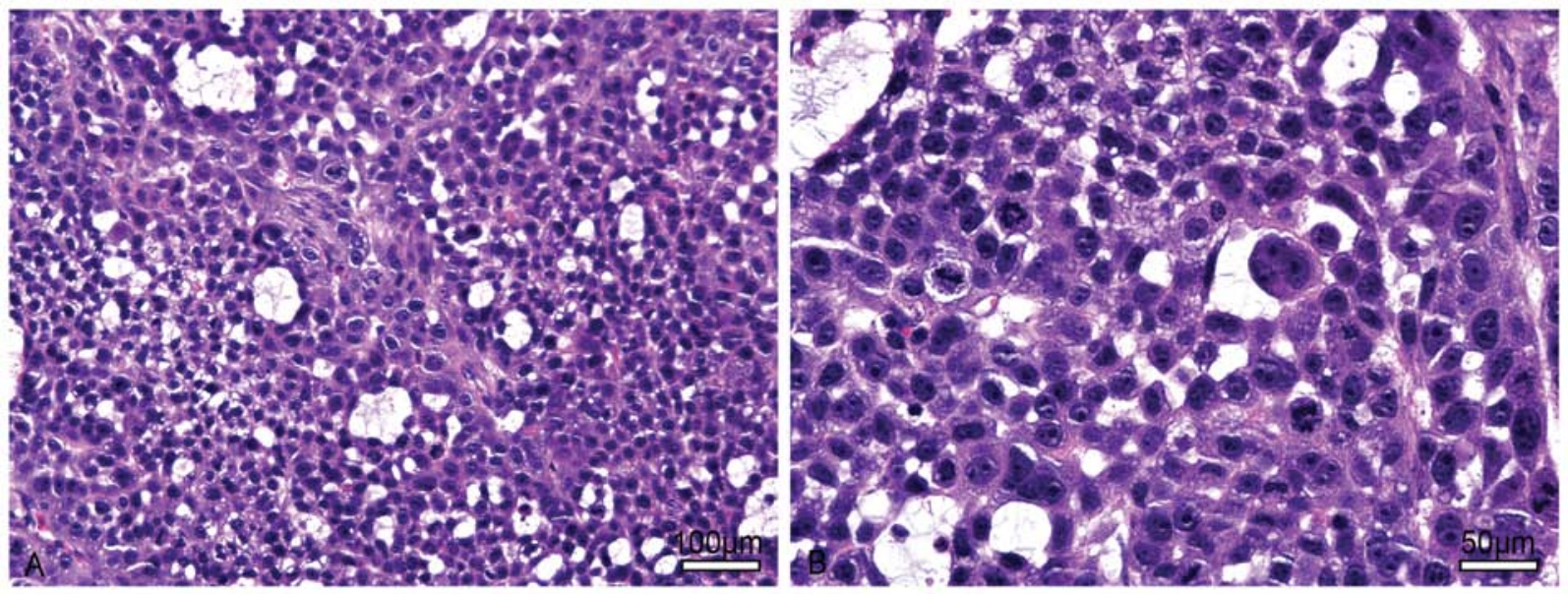

Figure 8. H\&E staining of the xenograft tumors. The tumor exhibits a solid pattern, and mucoid degeneration occurs in the local area. Tumor cells are round or oval and have rich cytoplasm and hyperchromatic nuclei. Mitosis and multinucleated tumor giant cells are common. (A) Original magnification, x200; scale bar, $100 \mu \mathrm{m}$. (B) Original magnification, $\mathrm{x} 400$; scale bar, $50 \mu \mathrm{m}$. 

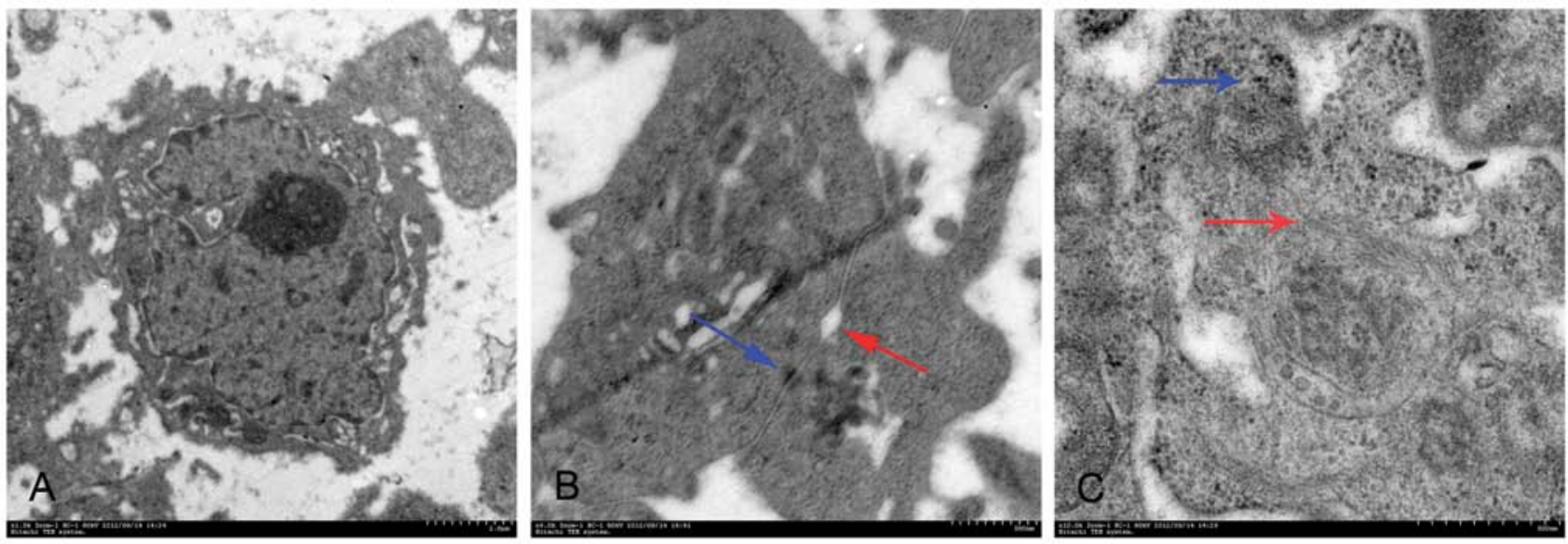

Figure 9. Morphograph of xenograft tumor cells under TEM observation. (A) Cytoplasm of the tumor cell is small, and the nuclei are large and markedly atypical. The nuclear membrane introcession and nucleoli are clear. Original magnification, $\mathrm{x} 1.5 \mathrm{k}$; scale bar, $2.0 \mu \mathrm{m}$. (B) Adjacent tumor cells are connected by a gap junction (red arrow). Some dysplasic desmosomes (blue arrow) appear. Original magnification, x6.0k; scale bar, $500 \mathrm{~nm}$. (C) Tonofilaments (red arrow) and many polyribosomes (blue arrow) are noted in the tumor cells. Original magnification, x10k; scale bar, $500 \mathrm{~nm}$.

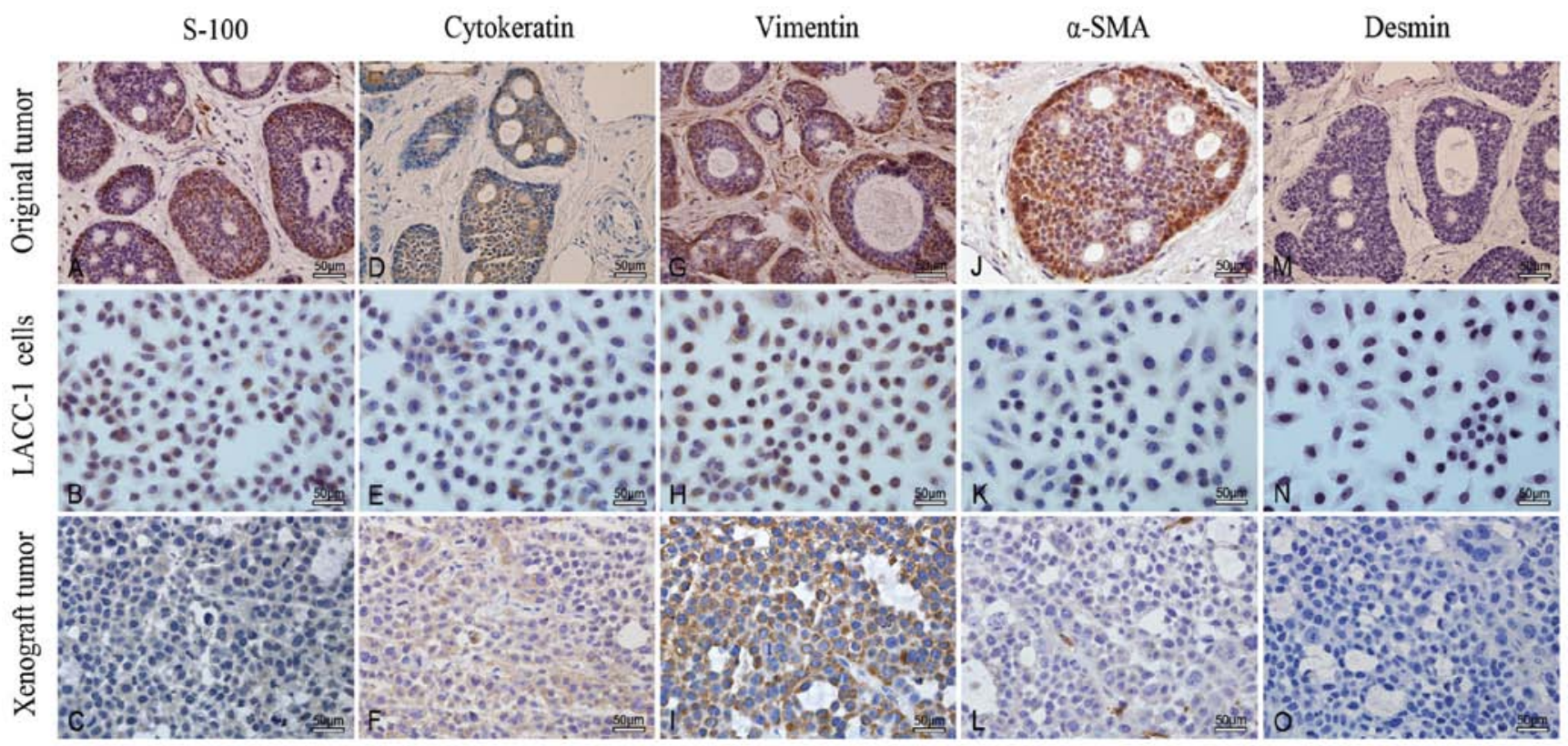

Figure 10. Immunocytochemistry for S-100, CK, vimentin, $\alpha$-SMA and desmin in the original tumor, LACC-1 cells and a xenograft tumor. (A-C) S-100 protein was immunostained in all three samples. Positive S-100 staining as brown particles is mainly localized in the nuclei and cytoplasm of the original tumor cells. In LACC-1 cells and the xenograft tumor, S-100 immunostaining is observed in the cytoplasm. Immunoreactivity of S-100 is stronger in the original tumor than that in the other two samples. (D-F) CK is immunostained in all three samples. Positive CK staining as brown particles is mainly localized in the cytoplasm of tumor cells. Immunoreactivity of CK is weaker in the xenograft tumor than in the other two samples. (G-I) Vimentin is immunostained in all three samples. Positive vimentin staining as brown particles is mainly localized in the cytoplasm of the tumor cells. Immunoreactivity of vimentin is the same in the three samples. (J-L) $\alpha$-SMA is immunostained in all three samples. Positive $\alpha$-SMA staining as brown particles is mainly localized in the cytoplasm of the tumor cells. Immunoreactivity of $\alpha$-SMA is stronger in the original tumor than that in the other two samples. (M-O) Desmin is not immunostained in all three samples. Original magnification, all x400. Scale bar, $50 \mu \mathrm{m}$.

and S-100 expression in the original tumor, LACC-1 cells and xenograft tumors. Immunoreactivity of these proteins gradually decreased in the three tissues (Fig. 10 and Table I).

RT-PCR. RT-PCR analysis showed a strong positive reaction to the human $\beta$-actin gene and weak reaction with the mice $\beta$-actin gene in the xenograft tumors. It also showed a human $\beta$-actin gene band in the LACC- 1 cell line and a mouse $\beta$-actin gene band in the nude mouse livers (Fig. 11).

\section{Discussion}

ACC of the human lacrimal glands is generally considered to originate from the lacrimal duct epithelium. It is a highly malignant neoplasm capable of diffuse invasion into adjacent tissues and distant metastasis. To investigate the biological characteristics of lacrimal gland ACC, we established the LACC-1 cell line. Many ACC cell lines have been established, such as KOA-1 (5), KOA-1L3 (6), ACC2 (7), ACC3 (7), 
Table I. Immunocytochemistry of S-100, CK, vimentin, $\alpha$-SMA and desmin in the original tumor, LACC- 1 cells and xenograft tumors.

\begin{tabular}{lccc}
\hline Protein & $\begin{array}{c}\text { Original } \\
\text { tumor }\end{array}$ & $\begin{array}{c}\text { LACC-1 } \\
\text { cells }\end{array}$ & $\begin{array}{c}\text { Xenograft } \\
\text { tumors }\end{array}$ \\
\hline S-100 & ++ & + & + \\
Cytokeratin & +++ & +++ & + \\
Vimentin & +++ & +++ & +++ \\
$\alpha-S M A$ & +++ & + & + \\
Desmin & - & - & - \\
\hline
\end{tabular}

A

Human $\beta$-actin
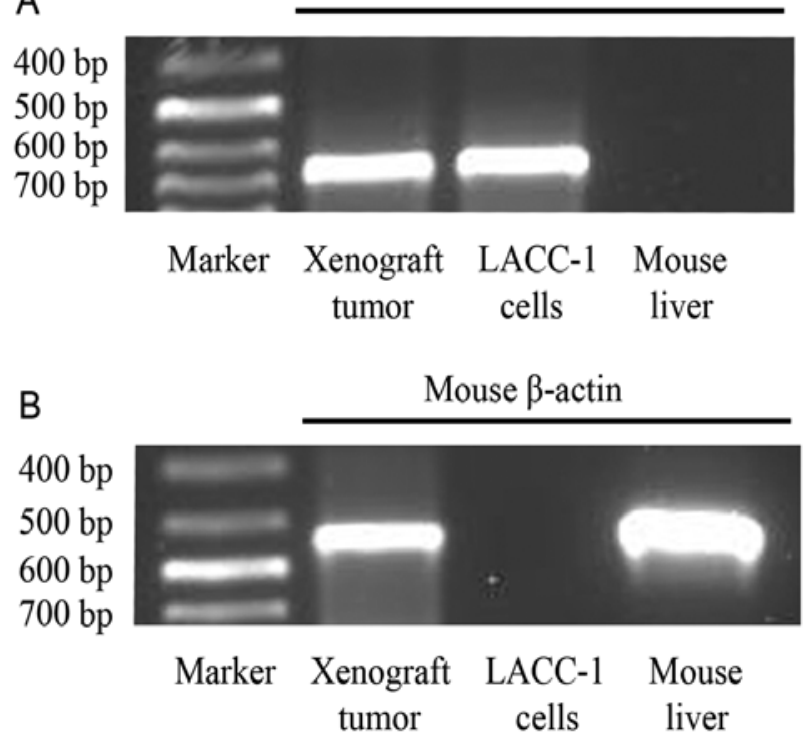

Figure 11. RNA expression levels of human and mouse $\beta$-actin in the xenograft tumors, LACC-1 cells and nude mouse liver. (A) Human $\beta$-actin amplification results. The size is $661 \mathrm{bp}$. Strong positive RNA expression level of human $\beta$-actin in the xenograft tumor and LACC-1 cells. Negative RNA expression level of human $\beta$-actin in the mouse liver. (B) Mouse $\beta$-actin amplification results. The size is $440 \mathrm{bp}$. Strong positive RNA expression level of mouse $\beta$-actin in mouse liver and its weak reaction in the xenograft tumor. Negative RNA expression level of mouse $\beta$-actin in the LACC-1 cells.

ACCS (8), ACCY (8), ACC-M (9), ACCNS (10), ACCI (11) and ACCIM (12). However, all these ACC cell lines were derived from the salivary glands. A recent study reports that sinonasal, lacrimal and tracheobronchial ACCs have significantly worse outcomes than ACC of the major salivary glands (13). Therefore, establishing a lacrimal gland ACC cell line is essential to study its unique biological behavior.

The most common cell primary culture methods are tissue block culture method and enzyme digestion. The former is simpler and more effective than the latter in solid tumor primary culture. Many ACC cell lines have been established through tissue block culture method, such as ACC2 (7), ACC3 (7), ACCS (8), ACCY (8) and SACC-83 (14).

To ensure the survival of isolated LACC-1 cells, a complete medium containing $20 \%$ FBS was used for the optimal growth.
At the same time, the cell culture flasks were pretreated with serum to promote tissue block adherence. At the early stage, tissue block adherence was not strong. Changing the medium required us to act slowly and gently. The fibroblasts predominated when the cells grew from the edge of the explants. To discard the fibroblasts and purified tumor cells, many different methods can be used. Tanaka et al (11) established the ACCNS cell line by culturing the cell mixture on a feeder layer of fibroblasts to remove the fibroblasts. Fibroblasts have a monolayer culture in a flask and are easy to dissociate, whereas epitheloid cells have a multilayer culture and are difficult to dissociate at the original stage. Thus, we used $0.25 \%$ trypsin and $0.02 \%$ EDTA mixture to detach the fibroblasts from the bottom of the flask while the epitheloid cells remained adhered to the flask. When the digestion procedure was stopped, the epitheloid tumor cells adhered to the flask faster and stronger than the fibroblasts. Thus, many fibroblasts were suspended and easily discarded. We used dissociation and repeated adherence methods to discard most of the fibroblasts and then combined continuous passage with the mechanical scraping method. Finally, we obtained pure epitheloid tumor cells after 8 passages. The malignant tumor characteristics then emerged.

LACC-1 cells appeared as a histologically solid pattern and continuous passage culture. They showed an epithelioid culture in the flask and presented with a cobblestone-like appearance when they reached confluency. The nuclei were large and round with many abnormal mitoses. The nucleoplasm ratio was high, and multinucleated giant cells were evident. LACC-1 cells showed a tendency to have overlapping growth without contact inhibition when the cell density continued to increase.

Under TEM, the LACC-1 cells were poorly differentiated. Mitosis and multinucleated giant tumor cells were common. The cytoplasm was small, whereas the cell nuclei were large and markedly atypical. The karyoplasmic ratio was reversed.

Many differences were noted between ACC-2 (7) and the LACC-1 cells under TEM. For example, in the LACC-1 cytoplasm, spherical zymogen secretory granules were found, and they had a uniform high-electron density. Large ribosomes appeared in the LACC-1 cells. The development of rough endoplasmic reticulum was not balanced in the different cells. Golgi complexes were occasionally visible. Other organelles were poorly developed. LACC-1 cells were connected by a tight junction instead of a typical desmosome junction.

The surface of the LACC-1 cells exhibited affluent microvilli, protuberances and filopodia under the SEM. This finding may be related to their high invasiveness and metastatic potential. When LACC-1 cell protuberances or filopodia adhere to adjacent connective tissues and nerves, they may increase basement membrane invasiveness and spread along the way or even invade blood vessels to cause hematogenous metastasis. The exact mechanisms of the invasive and metastatic ability of LACC-1 must be thoroughly studied further.

As detected by IHC, the original tumor, the LACC-1 cell line and the xenograft tumors expressed both CK and vimintin. CK is a key component of intermediate fibers of the cytoskeleton of epithelial cells and is expressed in epithelial cells, epithelial tumors and metastatic cells (15). Therefore, it is useful for identifying whether or not the metastatic tumor comes from the epithelium. Vimentin is an intermediate 
filament expressed in the tissues of normal mesenchymal origin. It is also a tumor biomarker for identifying a mesenchymal-derived tumor. However, it is known to be expressed aberrantly in epithelial cancers of the prostate, gastrointestinal tract, breast, central nervous system, lung and malignant melanomas (16). According to Chu et al (17), the coexpression of vimentin and $\mathrm{CK}$ intermediate filaments by epithelial or nonepithelial tumors is advantageous for migratory and invasive functions due to unique interactions between cell surface receptors, the cytoskeleton and ECM. Desmin is an intermediate filament protein that is widely distributed in skeletal muscle, cardiac muscle, smooth muscle and myoepithelial cells. However, it was not demonstrated in either the LACC-1 cell line or the original and xenograft tumors. The S-100 proteins are expressed in neural tissues and are involved in the regulation of cellular processes, such as cell cycle progression and differentiation. S-100 is localized in the cytoplasm and nuclei of astrocytes, Schwann cells, ependymomas and astrogliomas. Actin filaments can form both stable and labile structures and are crucial components of microvilli and the contractile apparatus of muscle cells. Furuse et al (18) found that the $\alpha$-smooth-muscle actin ( $\alpha$-SMA) is useful for the identification of myoepithelial cells, especially in cribriform and tubular ACC. In the present study, the original tumor, the LACC-1 cell line and xenograft tumors all showed a positive reaction to S-100 protein and $\alpha$-SMA. The LACC- 1 tumors had a perineural invasion biological feature. Under TEM, spherical zymogen secretory granules were observed in the LACC-1 cytoplasm. Thus, we speculated that the LACC-1 tumor could have neuroendocrine tumor characteristics.

To date, many heterotransplanted human ACCs have been established $(5,6,11,12,19)$, and the characterization of ACC proliferation, invasion and metastasis have been frequently reported. However, most of these xenograft model systems have been derived from the salivary glands or oral cavity. The present study first established a human ACC cell line of the lacrimal glands. The no. 84 generation LACC-1 cell line was inoculated subcutaneously into the subaxillary of nude mice. The tumorigenic potential was then evident. The formation rate of the transplanted tumors was $100 \%$ on day 7 after inoculation. This finding demonstrated that the LACC-1 cell line was malignant with tumorigenic ability. The xenograft tumors retained the same histological characteristics of a solid pattern as the LACC-1 original tumor after inoculation for 49 days. Lacrimal gland ACC usually exhibits a combination of three distinctive growth patterns: cribriform, tubular and solid. The predominant growth pattern is predictive of prognosis. For example, the solid pattern generally indicates worse prognosis when compared with the tubular or cribriform pattern.

RT-PCR was used to elucidate human xenograft tumors. A strong human $\beta$-actin gene band was detected in the PCR product and confirmed human origin. The weak mouse $\beta$-actin gene band remained probably because adjacent thin connective tissues covered the xenograft tumors and were combined with the tumors when total-RNA was extracted.

When the xenograft tumors were removed 49 days later, we observed that the tumors intruded into the chest wall and thoracic cavity of the mice instead of metastasizing to the lung, liver or axillary lymph nodes. In the clinic, ACCs derived from lacrimal glands have a different biological behavior than those derived from the salivary glands. For example, $\sim 40-60 \%$ of salivary gland ACCs develop distant metastases (i.e., lungs, bones and soft tissues) despite good local control of the tumor (20). Lacrimal gland ACCs may spread into the intracranial cavity, nasal cavity and temporal fossa or metastasize to the lung, bone, liver and lymph nodes. For lacrimal gland ACC, intracranial invasion is the leading cause of death, and the 5-year metastasis rate is $25.71 \%$ (9/35) (4), which is lower than that of the salivary glands. However, no metastatic phenomenon occurred during the 49 days possibly because the observation time was not long enough.

Overall, the newly established LACC-1 cell line was subcultured for more than 100 passages during the last two years. This cell line originated from a solid pattern of human lacrimal gland ACC and produced tumors through subcutaneous transplantation into nude mice. In conclusion, the LACC-1 cell line is the first established cell line derived from human lacrimal glands and to exhibit tumorigenicity in nude mice. The LACC-1 cell line provides a beneficial model for studying the biological characteristics of human lacrimal gland ACC.

\section{Acknowledgements}

The present study was supported by a grant from the National Natural Science Foundation of China (no. 31100991).

\section{References}

1. Lee DA, Campbell RJ, Waller RR and Ilstrup DM: A clinicopathologic study of primary adenoid cystic carcinoma of the lacrimal gland. Ophthalmology 92: 128-134, 1985.

2. Font RL and Gamel JW: Epithelial tumors of the lacrimal gland: an analysis of 265 cases. In: Ocular and Adnexal Tumors. Jakobiec FA (ed). Aesculapius, Birmingham, AL, pp787-805, 1978.

3. Font RL, Smith SL and Bryan RG: Malignant epithelial tumors of the lacrimal gland; a clinicopathologic study of 21 cases. Arch Ophthalmol 116: 613-616, 1998.

4. Lin TT, He YJ, Zhang H, Song GX, Tang DR and Zhao HF: Analysis of treatment and prognosis of orbital adenoid cystic carcinoma. Zhonghua Yan Ke Za Zhi 45: 309-313, 2009 (In Chinese).

5. Umeda M, Komatsubara H, Nishimatsu N, Oku N, Shibuya Y, Yokoo S and Komori T: Establishment and characterization of a human adenoid cystic carcinoma line of the salivary gland which is serially transplantable and spontaneously metastasises to the lung in nude mice. Oral Oncol 38: 30-34, 2002.

6. Umeda M, Komatsubara H, Ojima Y, Minamikawa T, Shibuya Y, Yokoo $\mathrm{S}$ and Komori T: Establishment and characterization of metastasizing cell lines from a heterotransplanted human adenoid cystic carcinoma. Oral Surg Oral Med Oral Pathol Oral Radiol Endod 98: 211-216, 2004.

7. He RG, Zhang XS, Zhou XJ, et al: The establishment of cell lines of adenoid cystic carcinoma of human salivary glands (ACC2, ACC3) and a study of morphology. West Chin J Stomatol 6: 1-4, 1988.

8. Shirasuna K, Watatani K, Furusawa H, Saka M, Morioka S, Yoshioka $\mathrm{H}$ and Matsuya T: Biological characterization of pseudocyst-forming cell lines from human adenoid cystic carcinoma of minor salivary gland origin. Cancer Res 50: 4139-4145, 1990.

9. Guan X, Qiu W and He R: The selection of highly lung metastatic salivary adenoid cystic carcinoma clone. Zhonghua Kou Qiang Yi Xue Za Zhi 31: 74-77, 1996 (In Chinese).

10. Guan XF, Qiu WL, He RG and Zhou XJ: Selection of adenoid cystic carcinoma cell clone highly metastatic to the lung: an experimental study. Int J Oral Maxillofac Surg 26: 116-119, 1997.

11. Tanaka N, Urabe K, Hashitani S, Sakurai K and Urade M: Establishment and characterization of a human adenoid cystic carcinoma cell line forming colonies cultured in collagen gel and transplantable in nude mice. Oncol Rep 17: 335-340, 2007. 
12. Hashitani S, Urade M, Zushi Y, Segawa E, Okui S and Sakurai K: Establishment of nude mouse transplantable model of a human adenoid cystic carcinoma of the oral floor showing metastasis to the lymph node and lung. Oncol Rep 17: 67-72, 2007.

13. Lin YC, Chen KC, Lin CH, Kuo KT, Ko JY and Hong RL: Clinicopathological features of salivary and non-salivary adenoid cystic carcinomas. Int J Oral Maxillofac Surg 41: 354-360, 2012.

14. Li SL, Liu XP and Zhang KH: Establishment of a human cancer cell line from adenoid cystic carcinoma of the minor salivary gland. Zhonghua Kou Qiang Yi Xue Za Zhi 25: 29-31, 62, 1990 (In Chinese).

15. Zhang X, Xie J, Yu C, Yan L and Yang Z: mRNA expression of CK19, EGFR and LUNX in patients with lung cancer micrometastasis. Exp Ther Med 7: 360-364, 2014.

16. Satelli A and Li S: Vimentin in cancer and its potential as a molecular target for cancer therapy. Cell Mol Life Sci 68 : 3033-3046, 2011.
17. Chu YW, Seftor EA, Romer LH and Hendrix MJ: Experimental coexpression of vimentin and keratin intermediate filaments in human melanoma cells augments motility. Am J Pathol 148: 63-69, 1996.

18. Furuse C, Sousa SO, Nunes FD, Magalhães MH and Araújo VC: Myoepithelial cell markers in salivary gland neoplasms.Int J Surg Pathol 13: 57-65, 2005.

19. Moskaluk CA1, Baras AS, Mancuso SA, Fan H, Davidson RJ, Dirks DC, Golden WL and Frierson HF Jr: Development and characterization of xenograft model systems for adenoid cystic carcinoma. Lab Invest 91: 1480-1490, 2011.

20. Ellis GL and Auclair PL: Tumors of the salivary glands. In: Atlas of Tumor Pathology. 3rd edition. Armed Forces Institute of Pathology, Bethesda, MD, pp203-216, 1996. 